\title{
Setting Hover, Roll, Yaw Pitch And Altitude Tricopter Using Arduino Multiwii
}

\author{
Yudhi Gunardi ${ }^{\mathrm{a},}$, , Hermawanysah ${ }^{\mathrm{a}}$ and Jumadril $\mathrm{JN}^{\mathrm{b}}$ \\ ${ }^{a}$ Universitas Mercu Buana, Jakarta, Indonesia \\ ${ }^{b}$ Universitas Batam, Batam, Indonesia
}

\begin{abstract}
The development and advancement of drone technology will be a trend now and in the future, it will have an important role as a material for mapping and aerial photography. with the use of Aeromodelling is a miniature model of an airplane, in which there are many types of aeromodelling, one of which is a tricopter, which is a drone driven by three motors that can be controlled by remote control using a remote control and has a Y-shaped frame. Many Drones are sold with easy-to-operate use but what if we assemble drones will be a lot of constraints because of many hardware and software settings in the manufacture of drones and this becomes a challenge in itself therefore This research specializes in hardware settings using software applications Multiwii Configuration Tools to set PID values for hover, roll, yaw pitch, and altitude values. So the Tricoptrer can go up and down, flight stability, and turn left and right movements. The conclusion of this research Tricopter can fly and move well following the desire to control the movement of tricoppter using a remote controller. The best tricopter flying navigation setting values obtained for Roll are $P=5.9, I=0.003, D=16$. Pitch is $P=5.7, I=0.003, D=17$. Yaw is $P=6.8, I=0.045, D=7$ the height is $P=6.4, I=0.025$, $D=13$.
\end{abstract}

Keywords: Tricopter, Multiwii, Sensor, Roll, Pitch, Yaw.

\section{Introduction}

Drone or often called drones is a new technology that is currently developing rapidly in the world. The drone is widely developed in the field of Military, mapping, research, photography [1-4]. The advantage of this technology can be used in dangerous places and missions without endangering the pilot. The drone currently is divided into two categories namely fixed wings (unmanned aircraft using wings) and multirotor (unmanned aircraft using more than one motor and without wings). This multirotor category usually uses 3 motors (tricopter), 4 motors (quadcopter), 6 motors (hexacopter), and 8 motors (octa copter).

Many Drones are sold with easy-to-operate use but what if we assemble drones will be a lot of constraints because of many hardware and software settings in the manufacture of drones and this becomes a challenge in itself therefore This research specializes in hardware settings using software applications Multiwii Configuration Tools to set PID values for hover, roll, yaw pitch, and altitude values. So the Tricoptrer can go up and down, flight stability, and turn left and right movements.

Tricopter is one type of drone that has a design shape such as a sign $(\mathrm{T})$ or $(\mathrm{Y})$ which later each end of the side will be installed a brushless DC motor [5-6], in this research, the tricopter to be

\footnotetext{
* Corresponding author. Tel.: +62-089668923090

E-mail address: yudhi.gunardi@mercubuana.ac.id

Manuscript History:

Received 25 November, 2020, Revised 10 January, 2021, Accepted 9 April, 2021, Published 30 April, 2021
} 
designed is a tricopter with $\mathrm{Y}$ shape design that can fly using three motors and propellers arranged horizontal triangle to air. So it has efficiency in the use of resources in flying navigation. Problems will arise namely how to design the value of Hover, Roll, Pitch, and Yaw. Therefore this research will solve this problem by using the Multiwii Configuration Tools software, which is expected to help design and fly a tricopter.

\section{System Architecture}

System Architecture's design of the tricopter is strongly influenced by the stability of hardware and software that will be described in the sub-chapter below.

\subsection{Tricopter}

A tricoptrer is an unmanned aircraft driven by three motors that can be controlled with a remote control or an airplane without a crew that controls inside. There are two main variations in unmanned aircraft control, the first variation is controlled by remote control and the second variation is an aircraft that is flying independently based on the program that is inputted to the aircraft before flying. The Tricopter itself is capable of carrying cameras, sensors, communication devices, and some other equipment.

\subsection{Control system}

The system design is divided into 2 parts, namely the signal sender and receiver of the signal as shown in Figure 1 below:

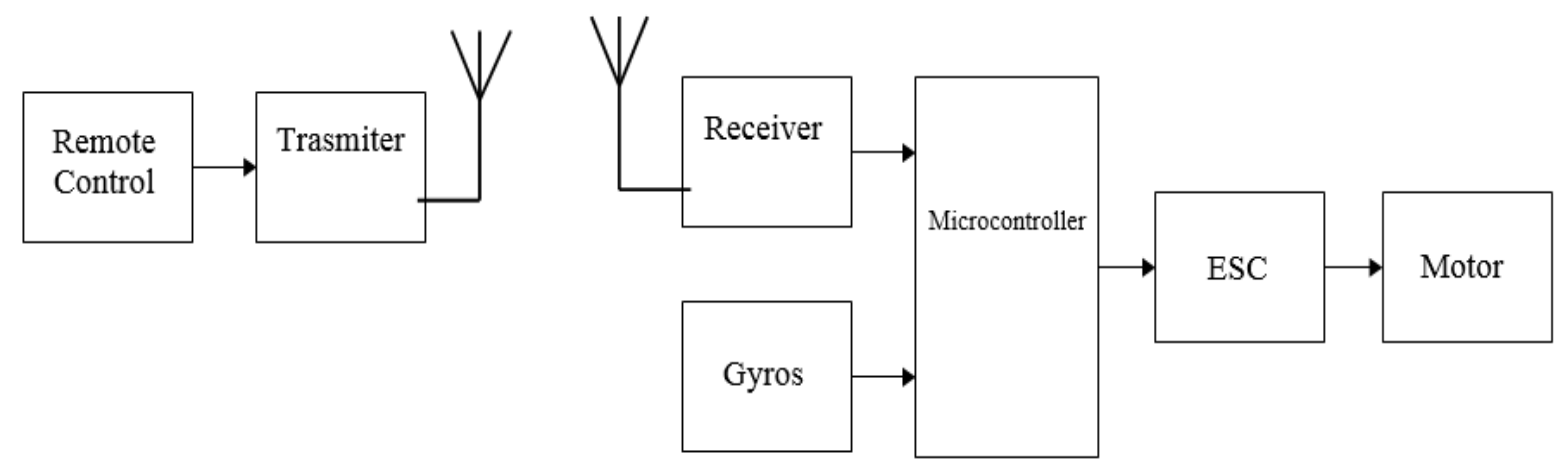

Figure 1. Diagram of a tricopter receiver and sender block

This radio control receiver circuit serves to receive signals sent by radio control for tricopter navigation systems which are then processed in multi-control. After the pins inserted into the multicontrol are connected, then connect the output pins of the multi-control to ESC and servo. The output pin of the microcontroller must be in accordance with the program created because in the program the pin will be determined as the output of the multi-control.

Microwii multi-controller is an electronic circuit board used in tricopter control, which is used in Arduino systems that have many uses such as medical fields [7], robot control [8-9]. Wireless control [10], safety engineering [11], On this board, there are various components which are important components in multicopter control.

Control of DC motor rotational speed can be done by adjusting the motor terminal voltage. ESC or Electric Speed Control is an electronic component that functions to control motor speed. The 
workings of this ESC are to receive pulse width from the microcontroller output which is then converted to regulate motor speed.

Brushless DC motors are developed from conventional brushed DC motors of separate excitation types that have the advantages of high efficiency, long life, low energy consumption and does not cause electrical noise.

\subsection{Mechanical Design}

Mechanical design and implementation of the tricopter, which is designed to be divided into three parts, including motor 1 , motor 2 , and motor 3 with a microprocessor controller placed in the middle of the support as shown in Figure 2.

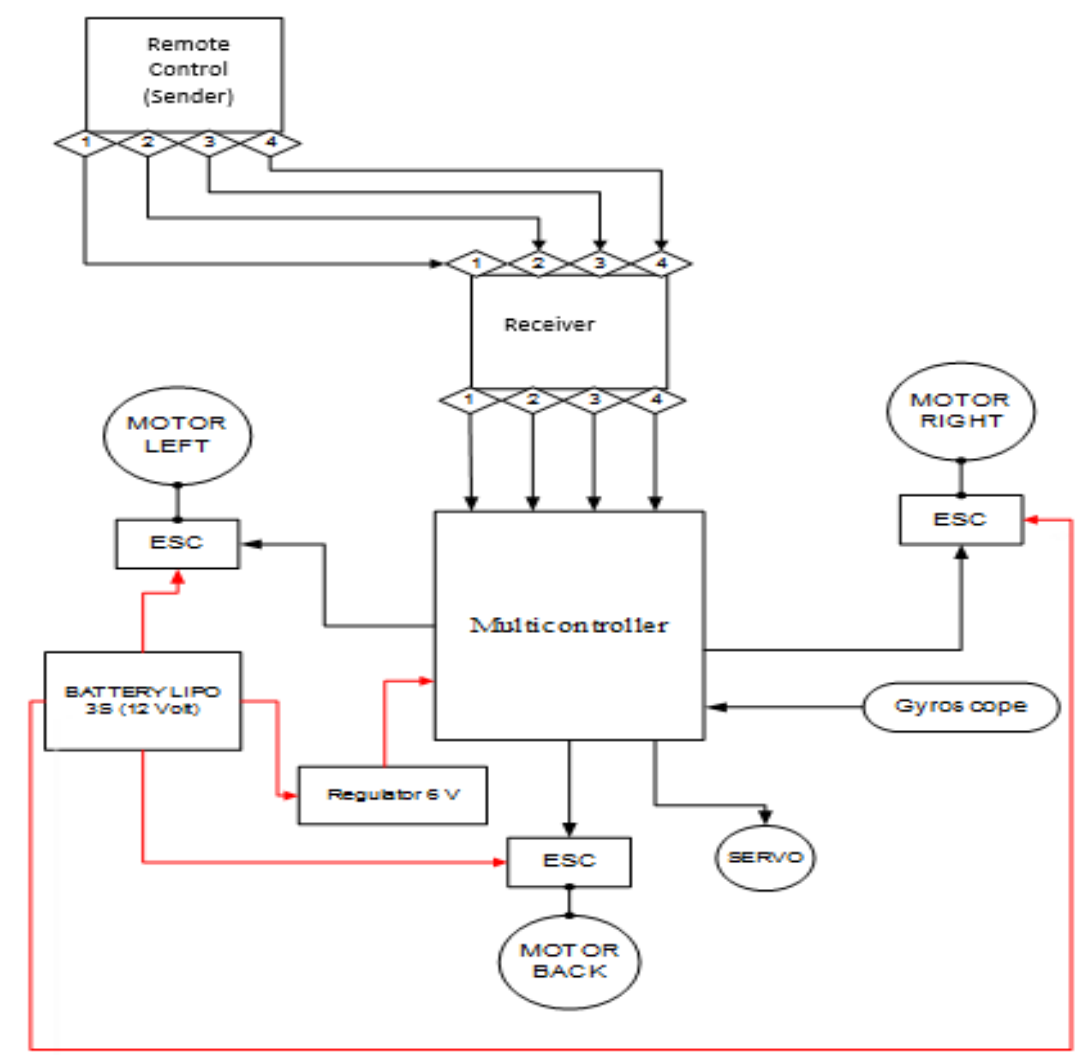

Figure 2. Block diagram

The battery functions as a voltage source for a multi-control circuit and ESC. Radio control is a tricopter navigation tool by sending frequency signals which are then received by the receiver.The receiver functions as a signal receiver that contains navigation commands for the tricopter of the radio control transmitter. Microwii functions as a control system of all tricopter navigation controller circuits. ESC functions as a regulator of the voltage and current entering the brushless DC motor. The DC brushless motor functions as a booster or provides a lift for the tricopter to fly. Servo serves to change the direction of the tricopter. Gyroscope MPU6050 serves to maintain the horizontal stability of the tricopter when in the air. 
To realize the device to be tested, the overall system of Microwii-based tricopter simulation circuit controlled by radio control is shown in Figure 3.

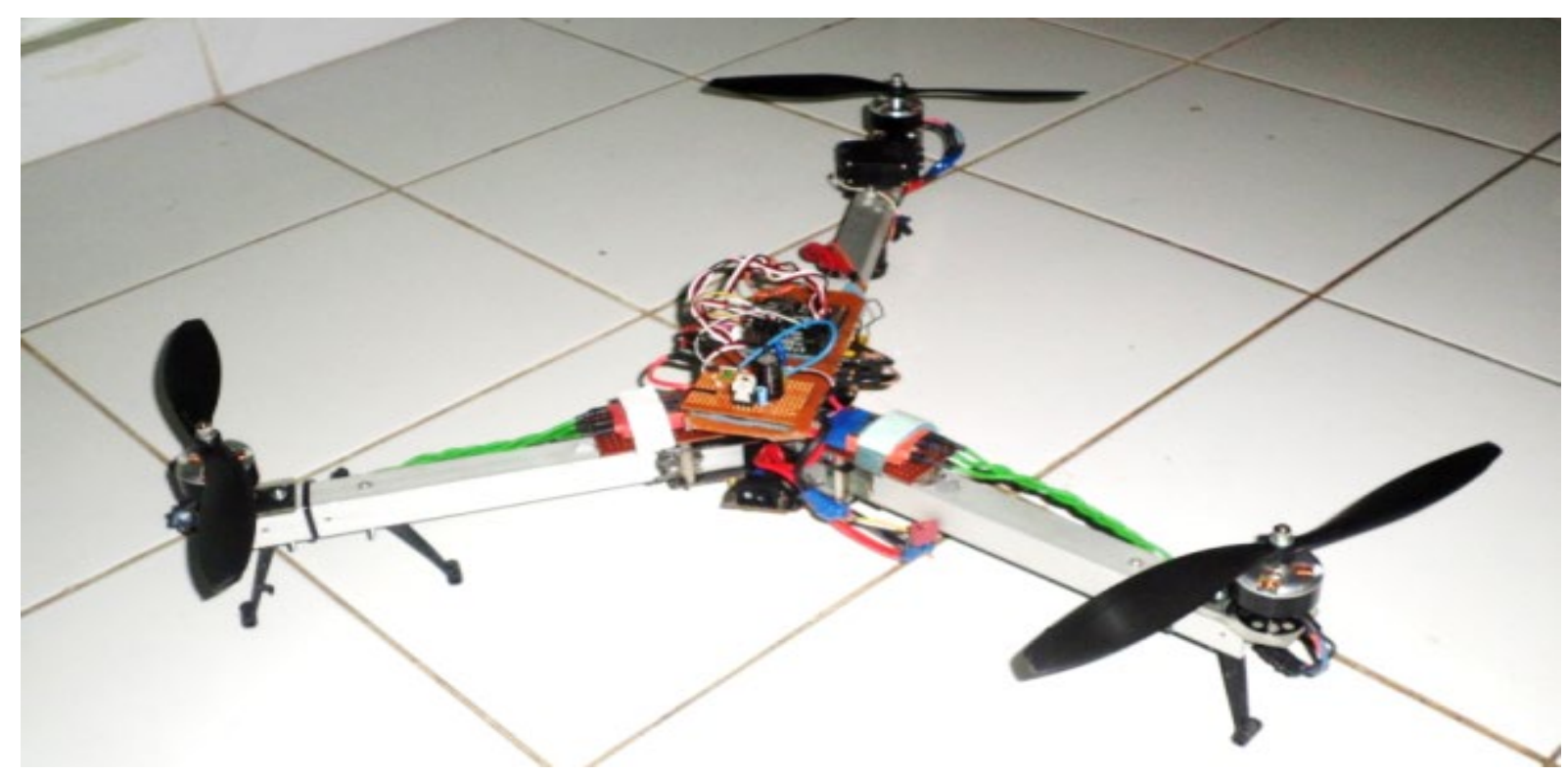

Figure 3. Mechanical design of the tricopter

The appearance of the Microwii module board is illustrated in Figure 4.

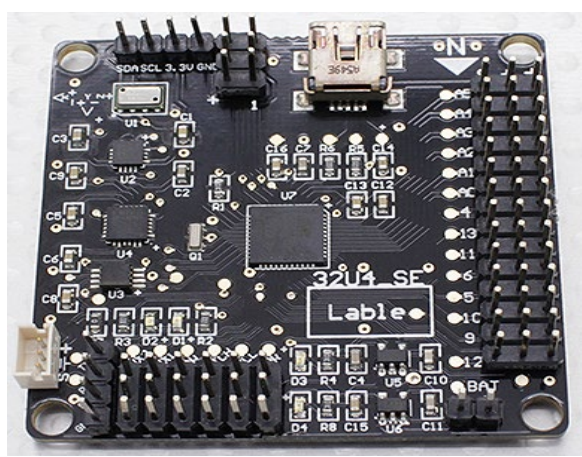

(A)

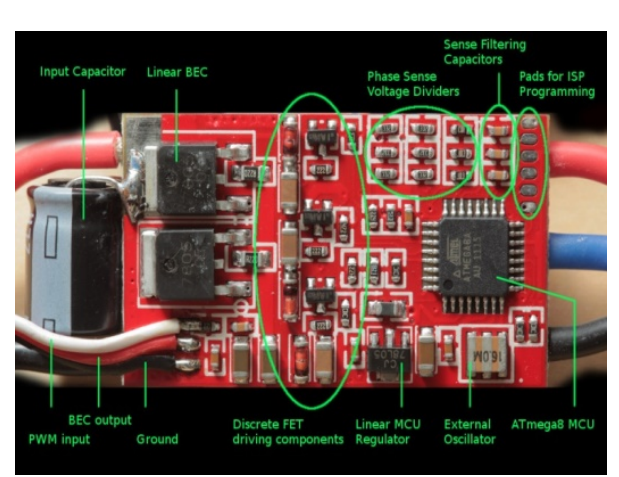

(B)

Figure 4. (A) Microwii board and (B) ESC 


\section{Navigation System}

The navigation system on the tricopter uses a multicontrolling dick radio. In this multicontroller, the signal output from the control radio is processed and used as an output to adjust the speed of the motor and move the servo. There are 3 kinds of navigation from the tricopter, namely Hover, Yaw, Pitch and Roll.

\subsection{Hover}

Hover is a tricopter that move up or down. The way the tricopter hovers is to move or rotate all three motors simultaneously. By the time all three motors move quickly, the tricopter will hover up. Conversely, if the speed of all three motors is low, then the tricopter will hover down. Figure 5 shows the hover movement of the tricopter.

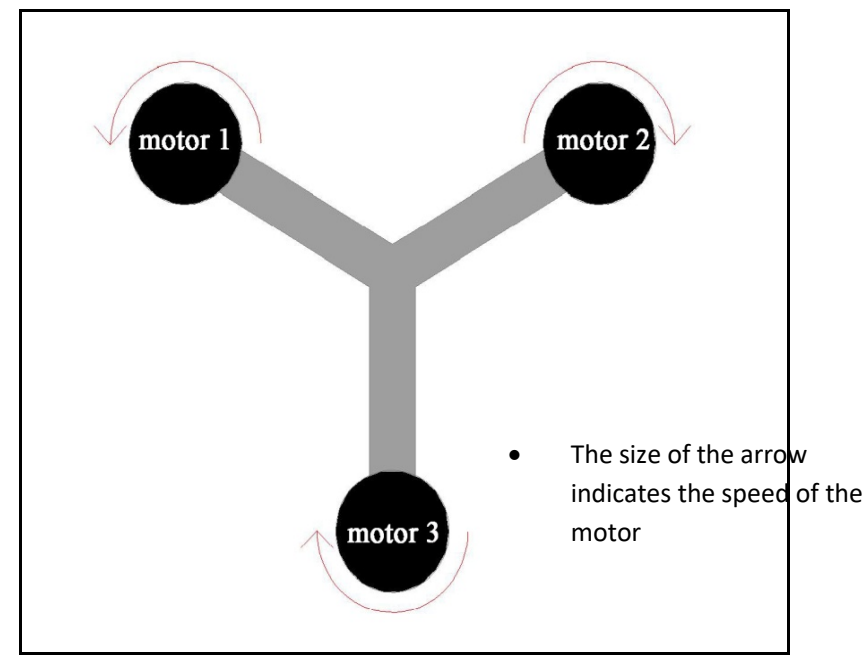

Figure 5. Tricopter hover move up

\subsection{Yaw}

Yaw is a tricopter movement based on the z-axis. The way yaw works are that the arm on the servo motor on the back moves up or down, then the arm changes the angle position on the rear motor to the right or left. If the servo arm goes up, then the tricopter will do yaw to the right, on the contrary, if the servo arm is down then the tricopter will do yaw to the left. figure 6 shows the working direction of the servo arm.

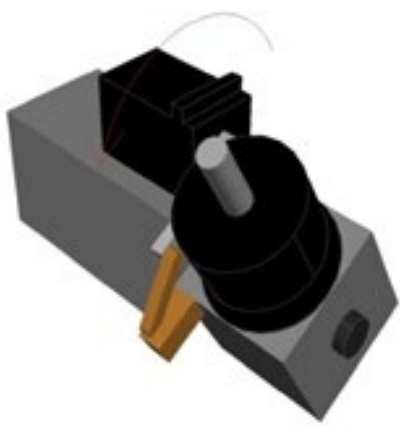

Figure 6. Tricopter yaw to the right of the motor servo down 


\subsection{Pitch}

Pitch is the movement of a tricopter based on the y-axis. It's this pitch that determines which tricopter moves forward or backwards. The pitch is determined by the rotation speed on the front and rear motors. If the motor pitches forward, the speed of the rear motor will be faster than the front motor, on the contrary if the speed of the rear motor is lower than the front motor. Move the pitch shown by figure 7 .

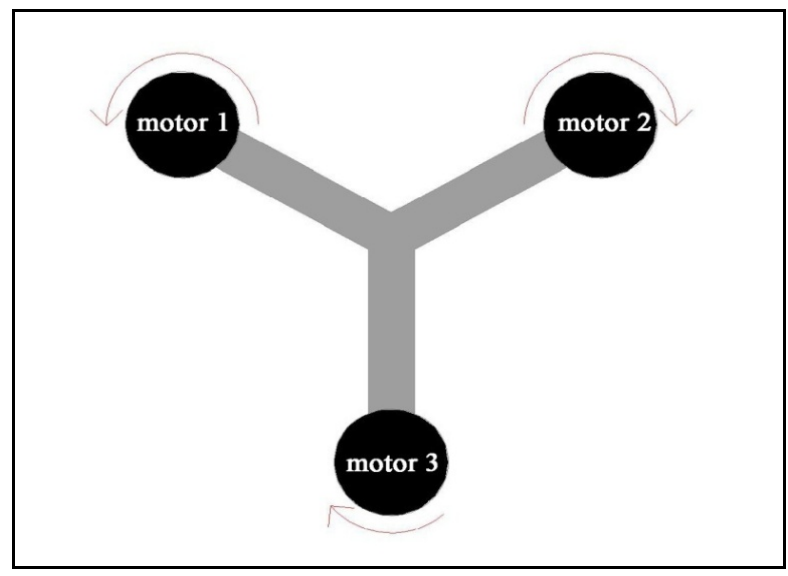

Figure 7. Tricopter pitch backwards

\subsection{Roll}

Roll is the movement of the tricopter based on the x-axis. The way the tricopter performs a roll move is determined by the speed of one of the front motors. If the right motor rotates faster, then the tricopter will roll to the left, on the contrary if the left motor is faster then the tricopter will roll to the right. Figure 8 shows the roll movement of the Tricopter.

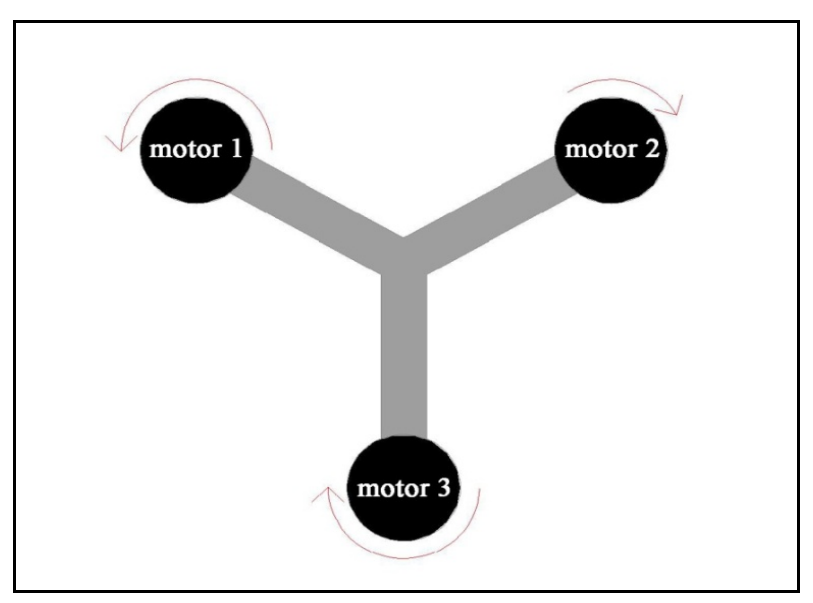

Figure 8. Tricopter roll to the right 


\subsection{Radio Control}

Radio control is an electronic device to control the machine remotely by utilizing radio waves. Each radio control system contains a transmitter (TX) and receiver (RX). For tricopters, the required number of movements is 4 types, therefore we need a radio control that has 4 or more channels.

Radio signals in the form of electrical pulses have unique codes for each command. The codes vary for flying, advancing, rotating, and other commands. This radio signal is then received by a receiver tucked in the tricopter. The code that is this command is sent to the circuit (Integrated Circuit or IC) in the tricopter. The code is then recognized and executed to drive the tricopter.

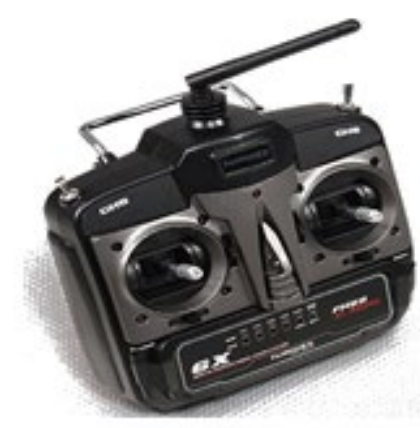

(a)

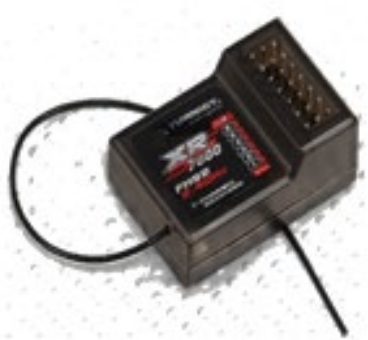

(b)

Figure 9. (a) Transmitter (TX), and (b) Receiver (RX)

\section{Testing and analysis}

The purpose of the overall system testing is to find out how the Tricopter moves and communicates in accordance with the controls performed by the user. Testing is done by trying the levers that are on the radio control transmitter. Every test is done Step-By-Step namely Hover, Roll, Pitch and Yaw using the application provided by Arduino

\subsection{Tricopter program application}

After the circuit is complete, the next step is to create a program in the Arduino IDE application. For the firmware program from the tricopter, use the Multiwii source code. Open the MultiWii.ino file in the multiwii folder as shown in Figure 5 and the Multiwii program display in Figure 6. 


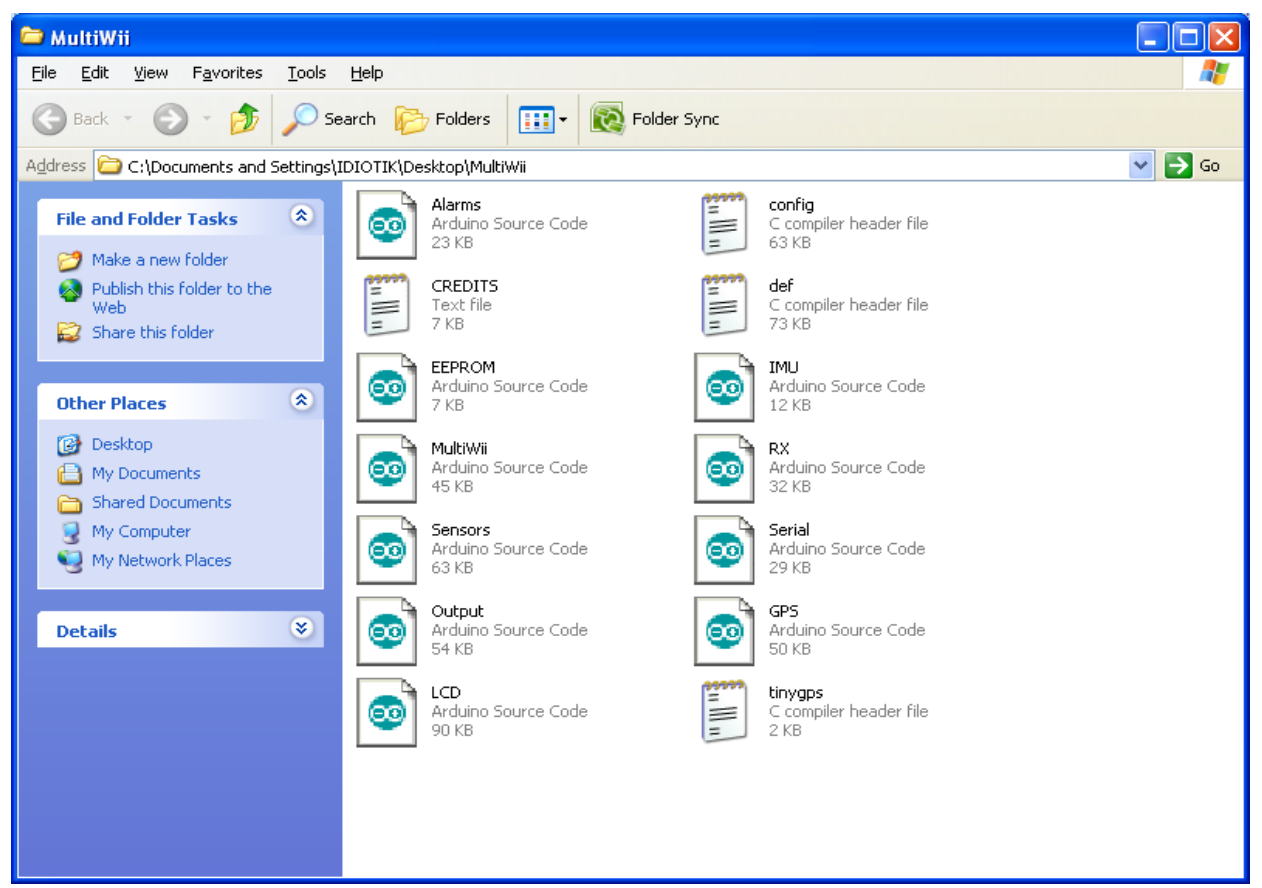

Figure 12. Multiwii folder

Multiwii Configurations Tools application. From this application, we can see the performance of programs and functions on the Microwii Board.

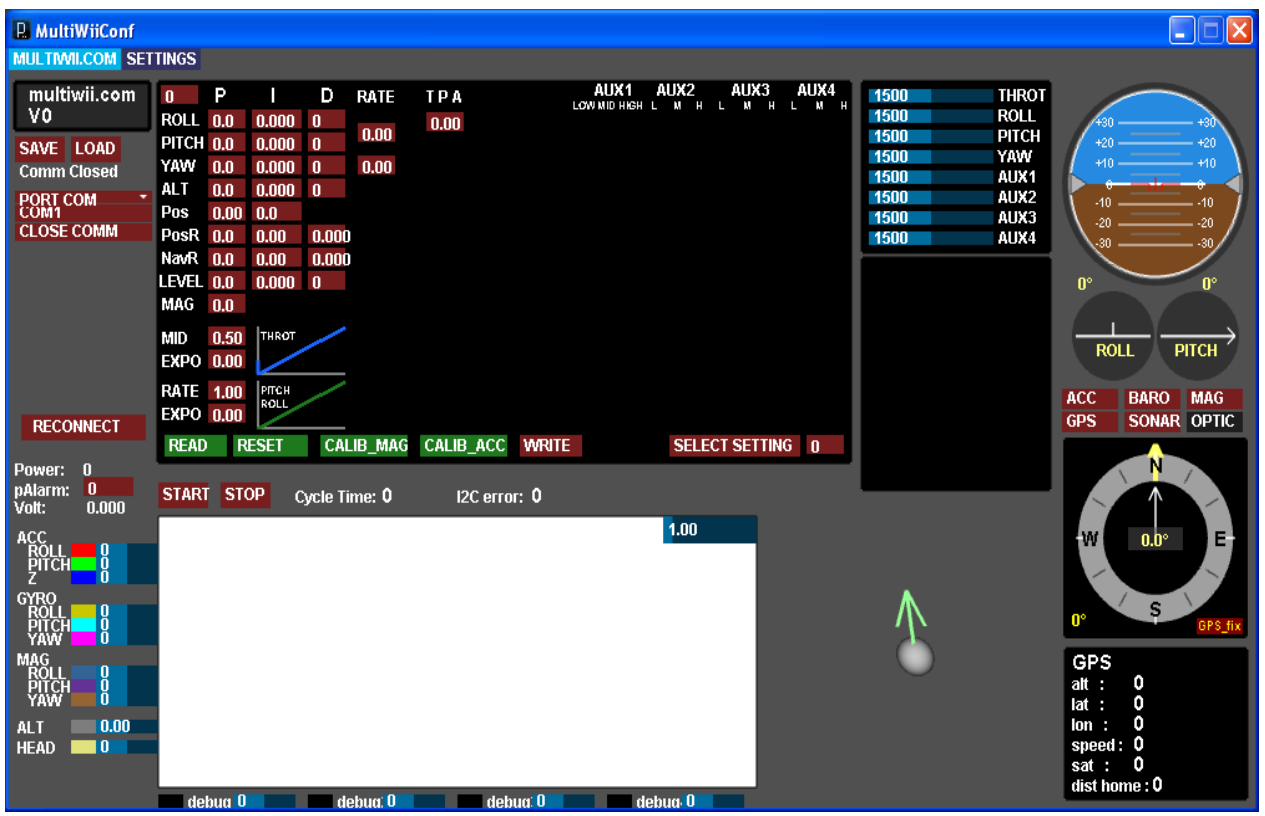

Figure 13. Multiwii configuration tools

To ensure the performance of the sensors on the Microwii Board, shake the Microwii Board. The graph will show irregular up and down lines, which indicates the Gyroscope and Accelerometer sensors are working properly. 


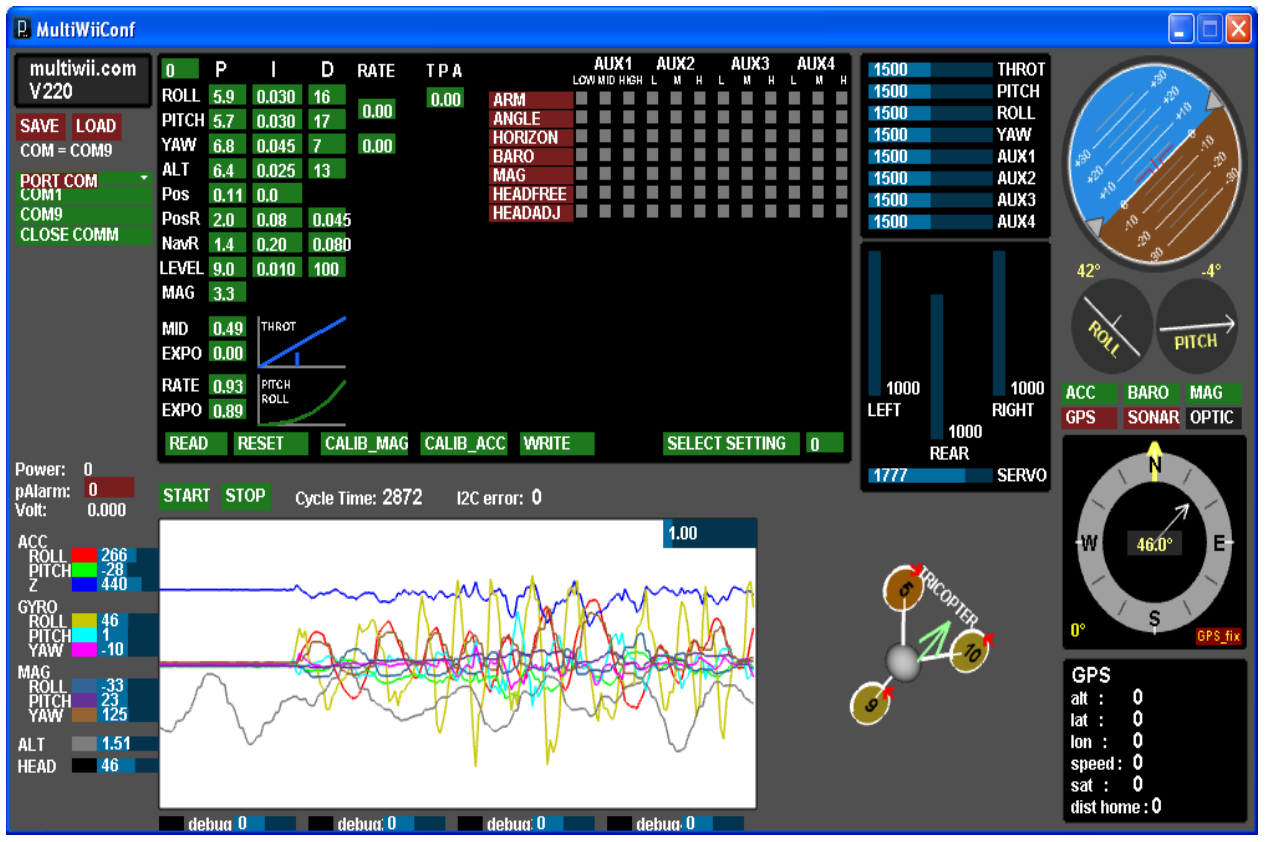

Figure 14. Sensor performance work on the Microwii board

The first test carried out is testing the movement of Hover, the purpose of this test is to show how the tricopter's movements when moving up and down.

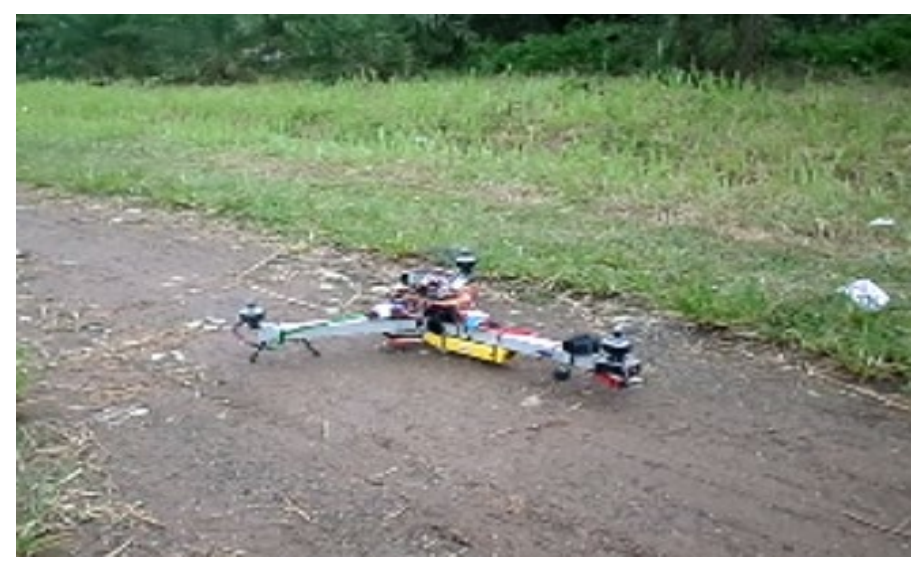

Figure 15. Tricopter flying preparation 


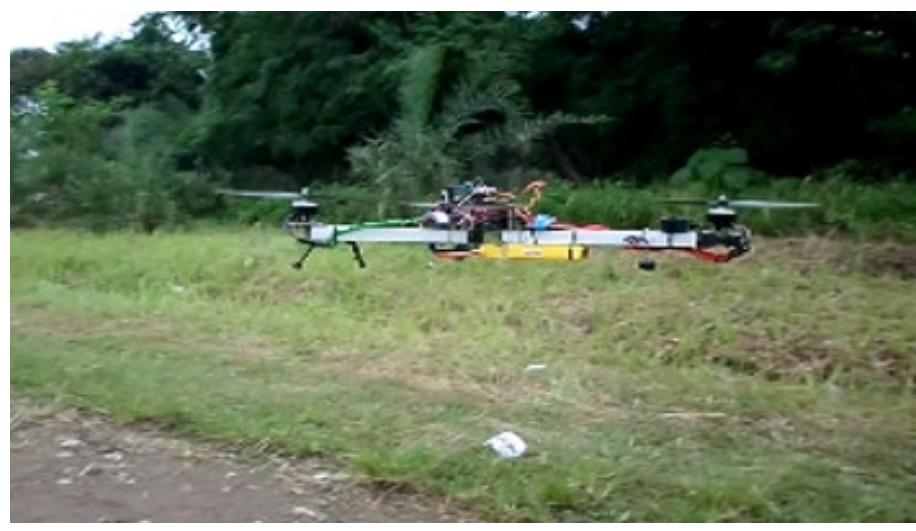

Figure 16. Tricopter hover rises

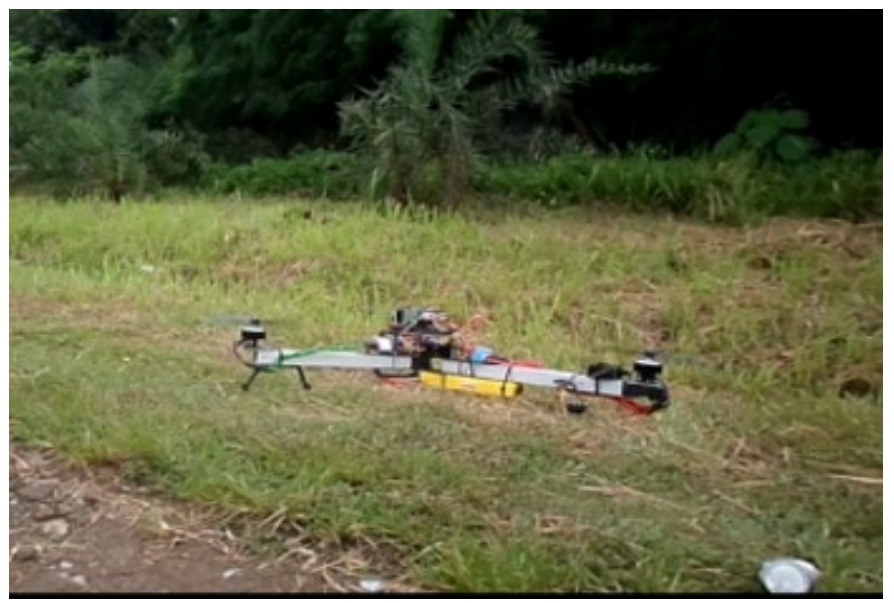

Figure 17. Tricopter moves down

From the picture, the results of the Hover's movement test went smoothly and the tricopter managed to move up and down according to orders.

\subsection{Roll Testing}

The second test is a roll movement test, the purpose of this test is to show how the tricopter moves when moving right and left. 


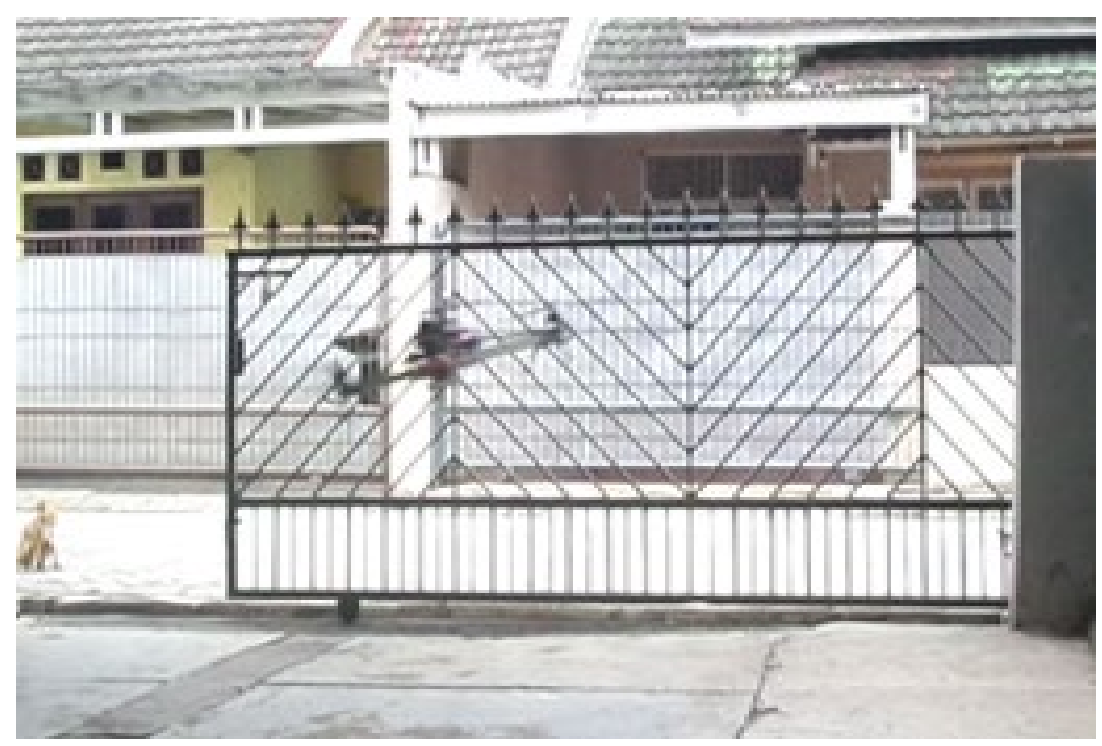

Figure 18. Roll movement to the left

From the picture, the results of this roll movement test went smoothly and the tricopter managed to move right and left according to the order.

\subsection{Yaw Testing}

The fourth test is a Yaw movement test, the purpose of this test is to show how the tricopter moves when it turns right and left.

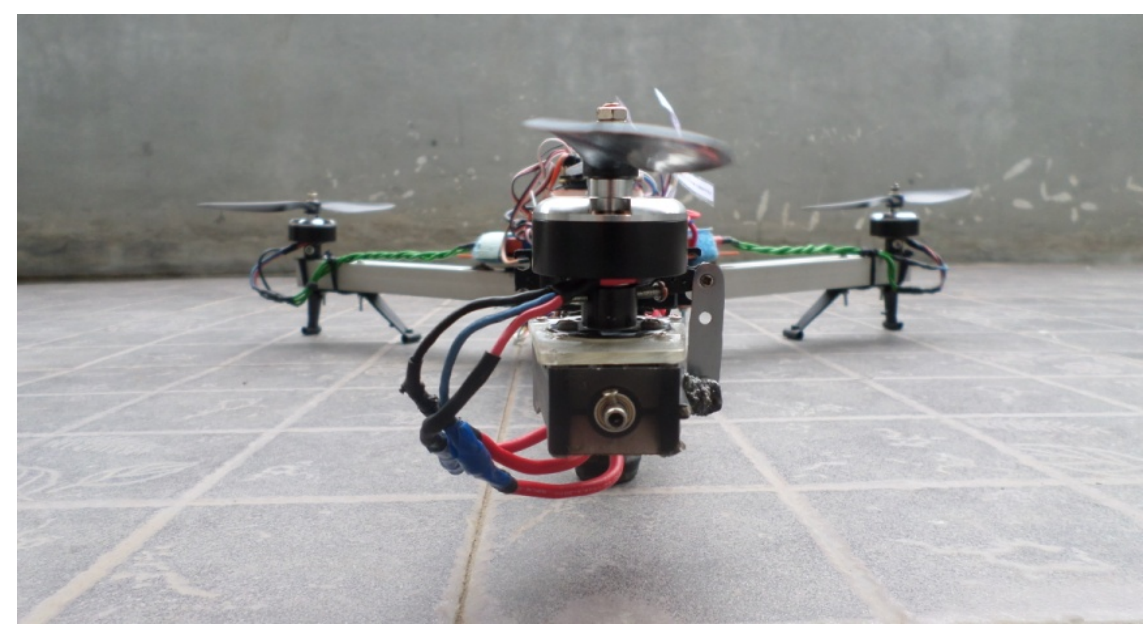

Figure 19. Servo position at rest 


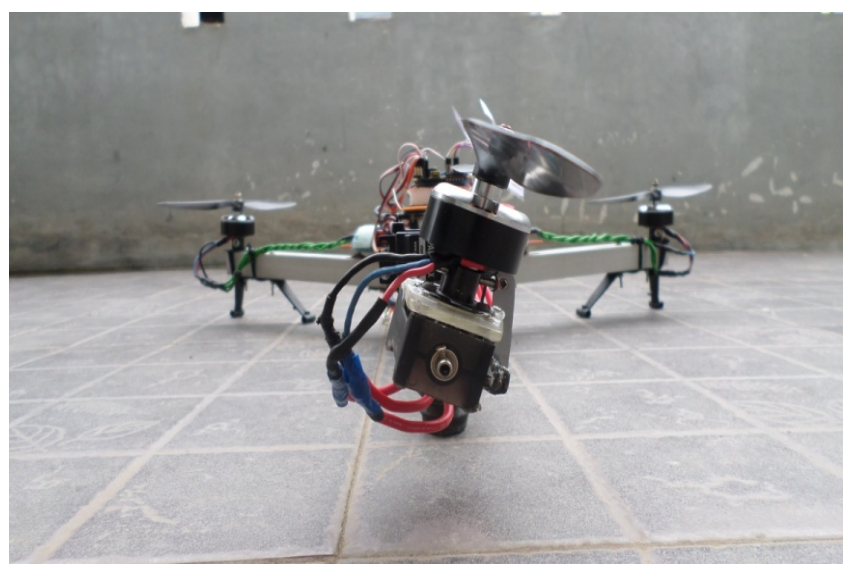

Figure 20. Servo position when Yaw left

From the picture, the results of this Yaw movement test went smoothly and the Tricopter managed to move to the left and right according to the command.

\subsection{Multiwii PID Settings}

After the Tricopter can work, the last step is setting the Multiwii PID using the Multiwii Configuration Tools. This stage serves to get the Tricopter stability value when flying. Open Multiwii Configuration Tools, select connect the program with Microwii Board.

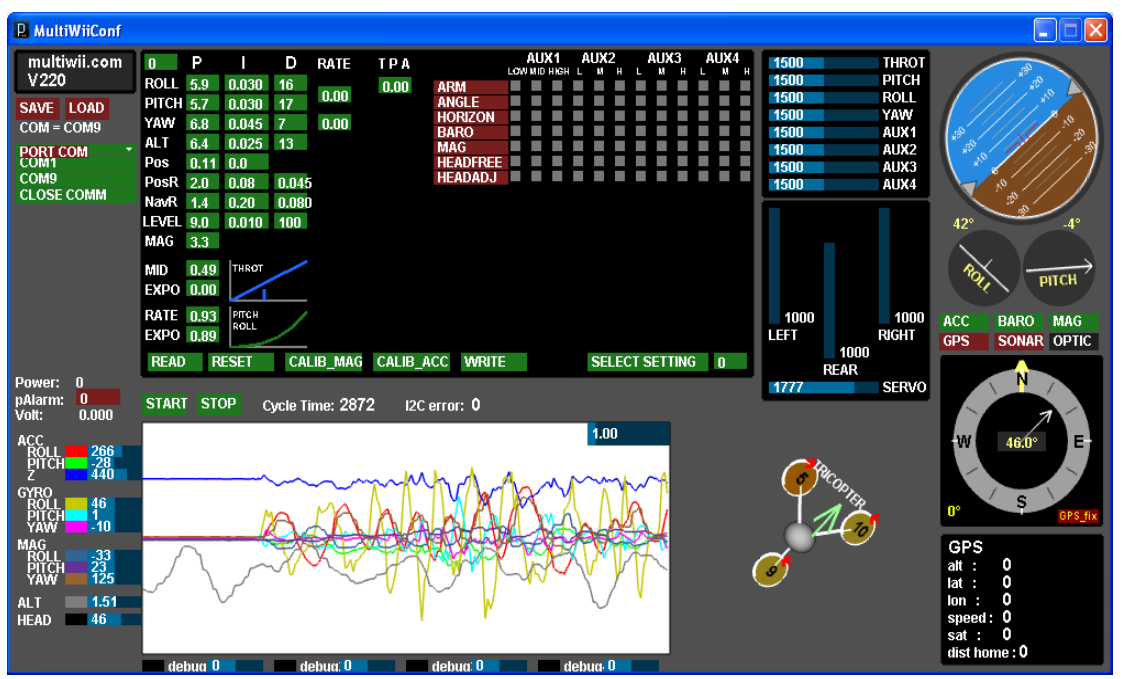

Figure 21. Microwii board connected with Multiwii GUI

The PID value search is carried out repeatedly until finding an accurate value. Accurate value is the value where the stable state of the multicopter when flying. This PID value is determined by the following factors:

a. The size, weight, type of material, and flexibility of the designed multi-copter.

b. Speed, lift, and power of the motor used.

c. Speed of sending data to the ESC.

d. Size and material of propellers

e. Pilot skills in balancing the fleet 
The first PID value setting is to determine the value of ROLL, PITCH, and YAW. This value serves to return the Tricopter to its original position before navigating. After that, determine the ALT (ALTITUDE) value, this value serves to balance the vertical motion of the Tricopter. After setting the PID repeatedly, the maximum value obtained from the PID Tricopter is as follows:

Table. 1 The best setting

\begin{tabular}{|c|c|c|c|c|}
\hline No & Navigation & P & I & D \\
\hline 1 & ROLL & 5,9 & 0,030 & 16 \\
\hline 2 & PITCH & 5,7 & 0,030 & 17 \\
\hline 3 & YAW & 6,8 & 0,045 & 7 \\
\hline 4 & ALT & 6,4 & 0,025 & 13 \\
\hline
\end{tabular}

\section{Conclusion}

It can be concluded that the tricopter from the assembly can fly well with hover, roll, pitch, yaw, alt. by setting the PID value on the Microwii Board. Control is carried out using radio control and can act according to instructions from the pilot. The best tricopter flying navigation setting values obtained for Roll are $\mathrm{P}=5.9, \mathrm{I}=0.003, \quad \mathrm{D}=16$. Pitch is $\mathrm{P}=5.7, \mathrm{I}=0.003, \mathrm{D}=17$. Yaw is $\mathrm{P}=6.8, \mathrm{I}=0.045, \mathrm{D}$ $=7$, the height is $\quad \mathrm{P}=6.4, \mathrm{I}=0.025, \mathrm{D}=13$.

\section{References}

[1] Hell, M., Bolam, R. C., Vagapov, Y., \& Anuchin, A. (2018). Design of a portable drone for educational purposes. In 2018 25th International Workshop on Electric Drives: Optimization in Control of Electric Drives (IWED), 1-5). , doi: 10.1109/IWED.2018.8321377

[2] Ebeid, E., Skriver, M., \& Jin, J. (2017, August). A survey on open-source flight control platforms of unmanned aerial vehicle. In 2017 Euromicro Conference on Digital System Design (DSD), 396-402. doi: 10.1109/DSD.2017.30.

[3] Song, Z., Li, K., Cai, Z., Wang, Y., \& Liu, N. (2016, August). Modeling and maneuvering control for tricopter based on the back-stepping method. In 2016 IEEE Chinese Guidance, Navigation and Control Conference (CGNCC), pp. 889-894. doi: 10.1109/CGNCC.2016.7828903.

[4] Jatsun, S., Emelyanova, O., Leon, A. S. M., \& Stykanyova, S. (2017, November). Control fligth of a UAV type tricopter with fuzzy logic controller. In 2017 Dynamics of Systems, Mechanisms and Machines (Dynamics), 1-5. doi: 10.1109/Dynamics.2017.8239459

[5] Bautista, J. A., Osorio, A., \& Lozano, R. (2017, June). Modeling and analysis of a tricopter/flying-wing convertible uav with tilt-rotors. In 2017 International Conference on Unmanned Aircraft Systems (ICUAS), 672-681, doi: 10.1109/ICUAS.2017.7991502.

[6] Ramp, M., \& Papadopoulos, E. (2015, September). On modeling and control of a holonomic vectoring tricopter. In 2015 IEEE/RSJ International Conference on Intelligent Robots and Systems (IROS), 662-668. doi: 10.1109/IROS.2015.7353443.

[7] Kadarina, T. M., \& Priambodo, R. (2017, November). Preliminary design of Internet of Things (IoT) application for supporting mother and child health program in Indonesia. In 2017 International Conference on Broadband Communication, Wireless Sensors and Powering (BCWSP), 1-6. doi: 10.1109/BCWSP.2017.8272576.

[8] Gunardi, Y., Hanafi, D., \& Supegina, F. (2018, October). Design of Navigation Mobile Robot Using Mirror Petri Net Method and Radio Frequency Identification. In 2018 Electrical Power, Electronics, Communications, Controls and Informatics Seminar (EECCIS) (pp. 102-107). doi: 10.1109/EECCIS.2018.8692926. 
[9] Gunardi, Y., Hanafi, D., Sulle, B., \& Supegina, F. (2019, July). Mathematics base for mobile robot navigation using mirror petri net Method. In Journal of Physics: Conference Series (Vol. 1230, No. 1, p. 012026). IOP Publishing.doi:10.1088/1742-6596/1230/1/012026

[10] Mahbub, M. (2019). Automated control signal reception acknowledgement system using nRF24L01P wireless transceiver module and Arduino. Journal of Applied Science \& Process Engineering, 6(1), 329339. https://doi.org/10.33736/jaspe.1333.2019

[11] Pranoto, H., Adriansyah, A., Feriyanto, D., Wahab, A., \& Zakaria, S. (2020). Propose safety engineering concept speed limiter and fatigue control using slifa for truck and bus. Sinergi, 24(3), 237-244. doi: http://dx.doi.org/10.22441/siner 